\title{
On the Universality of Chain Dynamics
}

\author{
K. L. Ngai, ${ }^{* \dagger}$ D. J. Plazek, ${ }^{\ddagger}$ and C. M. Roland ${ }^{*} \dagger$ \\ Naval Research Laboratory, Washington, D.C. 20375-5342, and Department of Material Science and \\ Engineering, University of Pittsburgh, Pittsburgh, Pennsylvania 15261
}

Received December 18, 2007; Revised Manuscript Received March 24, 2008

\begin{abstract}
On the basis of a comparison of the dielectric normal mode relaxation times $\tau_{\mathrm{n}}$ of unentangled cis-1,4-polyisoprene, poly(propylene glycol), and polyoxybutylene with literature data for the shear viscosity $\eta$ of polystyrene, atactic polypropylene, and polycarbonate from various sources, Ding and Sokolov [Macromolecules 2006, 39, 3322] concluded that the $T_{\mathrm{g}}$-normalized temperature dependence of $\tau_{\mathrm{n}}$ and $\eta$ is the same for all polymers. Liu et al. [Macromolecules 2006, 39, 8867] reached the same conclusion from an examination of their own dynamic mechanical modulus data for 1,4-polybutadiene, polyisobutylene, polystyrene, polycarbonate, and eight aliphatic copolymers. We verify that the data in these studies ostensibly support a universal $T_{\mathrm{g}}$-normalized temperature dependence; however, a detailed analysis of viscoelastic data on many different polymers, from creep compliance measurements carried out in the same laboratory, shows unambiguously that the $T_{\mathrm{g}}$-normalized temperature dependences of $\tau_{\mathrm{n}}$ and $\eta$ can vary substantially among polymers. Thus, the behavior of $\tau_{\mathrm{n}}$ and $\eta$ is not universal.
\end{abstract}

\section{Introduction}

Recently, Sokolov and co-workers ${ }^{1,2}$ compared the $T_{\mathrm{g}^{-}}$ normalized temperature dependence of the terminal, or normal mode, relaxation time, $\tau_{\mathrm{n}}$, of several high cis-1,4-polyisoprene (PI) samples with molecular weights $M=2.46$ and $21.2 \mathrm{kDa}$ from one study ${ }^{1}$ and 0.870 and $9.55 \mathrm{kDa}$ from another. ${ }^{2} T_{\mathrm{g}}$ was defined as the temperature at which the dielectric segmental relaxation time $\tau_{\alpha}$ equals $1 \mathrm{~s}$ in ref 1 and $10 \mathrm{~s}$ in ref 2 . After applying vertical shifts to plots of $\log \tau_{\mathrm{n}}$ vs $T_{\mathrm{g}} / T$, they found that the normal mode dielectric relaxation times of the lower and higher molecular weight PI superposed onto one master curve. This curve also included analogous dielectric and mechanical terminal relaxation data for other polymers taken from the literature: dielectric ${ }^{3,4} \tau_{\mathrm{n}}$ of polyoxybutylene (POB) with $M_{\mathrm{w}}=0.97$ and $0.46 \mathrm{kDa}$ and poly(propylene glycol) (PPG) with $M_{\mathrm{w}}=4.00 \mathrm{kDa}$, mechanical compliance determinations of the terminal relaxation time or viscosity of atactic polypropylene (aPP) $)^{5,6}$ and polystyrene (PS) ${ }^{7-9}$ from the laboratory of one of the authors, and the viscosity of polycarbonate (PC) obtained by an unusual technique in another laboratory. ${ }^{10}$ These findings from mixed dielectric relaxation and mechanical data led Sokolov and co-workers ${ }^{1,2}$ to suggest the existence of a universal normalized temperature dependence of the terminal relaxation time of polymers. Liu et al. ${ }^{11}$ examined their own dynamic mechanical modulus data for 1,4-polybutadiene (PB), PI, polyisobutylene (PIB), PS, PC, and eight aliphatic copolymers, together with creep compliance data for aPP., 5 The time-temperature shift factors, $a_{\mathrm{T}}$, for the terminal relaxation were plotted against $T_{\mathrm{g}} / T$ for each polymer, with $T_{\mathrm{g}}$ obtained from calorimetry. After applying vertical shifts, they showed that the $T_{\mathrm{g}}$-normalized temperature dependence of $\log a_{\mathrm{T}}$ for the polymers fall on one master curve. From this result Liu et al. ${ }^{11}$ concluded that the $T_{\mathrm{g}}$-normalized temperature dependence of the viscosity $\eta$ or the terminal relaxation time of amorphous polymers did indeed exhibit universality. In fact, whereas Sokolov et al. ${ }^{1,2}$ reported universal $T_{\mathrm{g}}$-scaled temperature dependence of $\tau_{\mathrm{n}}$ over the non-Arrhenius range of ca. $0.8<$ $T_{\mathrm{g}} / T<1$, Liu and co-workers ${ }^{11}$ claimed the same for a wider

\footnotetext{
* Corresponding author.

Naval Research Laboratory.

$\doteqdot$ University of Pittsburgh.
}

range down to $T_{\mathrm{g}} / T \sim 0.5$, which encompasses the regime of Arrhenius behavior.

If valid, a universal $T_{\mathrm{g}}$-normalized temperature dependence of $\tau_{\mathrm{n}}$ and $\eta$ for all polymers of any molecular weight (entangled or unentangled) would be a remarkable result, unanticipated by theories of polymer viscoelasticity. Therefore, it is necessary to critically examine the data and procedures used to arrive at this putative finding. First, the analyses ${ }^{1,2,11}$ relied on $T_{\mathrm{g}}$ values from various sources using different techniques and definitions. It is well-known that the apparent $T_{\mathrm{g}}$ of a polymer, and hence its $T_{\mathrm{g}}$-normalized dependences, can vary significantly depending on the measurement technique as well as the thermal history of the sample. ${ }^{12,13}$ Second, unlike transient creep measurements (the source of the data for $\mathrm{aPP}^{5,6}$ ), methods such as dielectric and dynamic mechanical spectroscopies cannot be used for direct determination of $\tau_{\mathrm{n}}$ or $\eta$ of high molecular weight polymers near $T_{\mathrm{g}}$ because the relaxation times become too long for these (relatively) high-frequency techniques. (Note that Sokolov et al. ${ }^{1,2}$ took $T_{\mathrm{g}}$ to be the temperature at which the dielectric segmental relaxation time $\tau_{\alpha} \leq 10 \mathrm{~s}$ because data were unavailable for the more customary $\tau_{\alpha}\left(T_{\mathrm{g}}\right)=100 \mathrm{~s}$; yet $\tau_{\mathrm{n}}$ are even larger than $\tau_{\alpha}$.) Thus, most of the dielectric and dynamic mechanical data analyzed in refs 1,2 , and 11 cannot provide values of $\tau_{\mathrm{n}}$ or $\eta$ near $T_{\mathrm{g}}$. Nevertheless, Liu et al. ${ }^{11}$ report (their figure 1) shift factors for the dynamic mechanical terminal relaxation times of high molecular weight PS at temperatures within $1 \%$ of the calorimetric $T_{\mathrm{g}}=105^{\circ} \mathrm{C}\left(T_{\mathrm{g}} / T \approx 0.99\right)$, which is surprising because of the experimental limitations of dynamic mechanical spectroscopy for extremely low frequencies. Part of the problem is the significant variations in the value of $T_{\mathrm{g}}$ as determined by different techniques and even with different cooling, $q_{\mathrm{c}}$, or heating, $q_{\mathrm{h}}$, rates for the same technique. ${ }^{12,13}$ For example, the calorimetric $T_{\mathrm{g}}$ of high molecular weight PS is $105{ }^{\circ} \mathrm{C}$ for heating at $10 \mathrm{deg} / \mathrm{min}$ after cooling at the same rate, but the dilatometric $T_{\mathrm{g}}$ is $98{ }^{\circ} \mathrm{C}$ for cooling at 1 $\mathrm{deg} / \mathrm{min} .^{12-14}$ Since the temperature sensitivity of $\tau_{\mathrm{n}}$ or $\eta$ is greatest close to $T_{\mathrm{g}}$, a critical test for a universal $T_{\mathrm{g}}$-normalized temperature dependence must be made using terminal relaxation data near $T_{\mathrm{g}}$.

There exist precise experimental data for a number of amorphous polymers that conform to the three requirements for critical assessment of $\eta\left(T_{\mathrm{g}} / T\right)$ or $\tau_{\mathrm{n}}\left(T_{\mathrm{g}} / T\right)$ universality: (i) consistency of the data by virtue of coming from one laboratory; 
(ii) uniformity in the definition and method of determination of $T_{\mathrm{g}}$; and most significantly (iii) the capability of obtaining $\eta$ or $\tau_{\mathrm{n}}$ sufficiently close to $T_{\mathrm{g}}$. These data, ${ }^{5,6,8,14-25}$ analyzed in the next section, were obtained by creep and recovery compliance measurements, a technique uniquely suited to characterizing the ultraslow dynamics of entangled polymers. We find that some polymers do exhibit equivalent $T_{\mathrm{g}}$-normalized temperature dependences of $\eta$ or $\tau_{\mathrm{n}}$; however, considering the polymers in toto, the results do not support the existence of a universality.

\section{2. $T_{\mathrm{g}}$-Normalized Terminal Relaxation Time from Shear Creep Measurements}

In this section we consider viscosities and terminal retardation times of amorphous polymers measured by creep and recoverable compliances. Note that although the terminal retardation times, $\tau_{\eta}$, from the compliance are longer than the (modulusderived) terminal relaxation time $\tau_{\mathrm{n}}$, their temperature dependences are the same. ${ }^{26}$

a. Polystyrene. Data of nearly monodisperse PS encompassing a broad range of molecular weights were reported by Plazek and O'Rourke. ${ }^{14}$ The nearly monodisperse samples considered in the present study (using the sample designations of ref 14) are $\mathrm{A} 67(M=1.1 \mathrm{kDa}), \operatorname{PC} 11(M=3.4 \mathrm{kDa})$, TAPS28 $(M=$ $12.7 \mathrm{kDa}), \mathrm{A}-61(M=16.4 \mathrm{kDa}), \mathrm{A}-25(M=47 \mathrm{kDa}), \mathrm{M} 102$ $(M=94 \mathrm{kDa}), \mathrm{L} 2(M=189 \mathrm{kDa})$, and A-19 $(M=600 \mathrm{kDa})$. From $\eta$ and the steady-state compliance $J_{\mathrm{s}}$ in Table II therein ${ }^{14}$ (the designation $J_{\mathrm{e}}$ was used instead of $J_{\mathrm{s}}$ ), the terminal retardation times are calculated as a function of temperature according to ${ }^{27}$

$$
\tau_{\eta}=\eta J_{\mathrm{s}}
$$

For high molecular weight PS, $J_{\mathrm{s}}$ is effectively temperature independent, and hence $\tau_{\eta}$ and $\eta$ have the same $T$-dependence. However, for low molecular weight unentangled polymers, $J_{\mathrm{s}}$ can decrease substantially with temperature upon approach to $T_{\mathrm{g}}{ }^{14,15,28}$ This well-known ${ }^{14,15}$ effect is due ${ }^{22}$ to the local segmental relaxation time $\tau_{\alpha}$ having a stronger temperature dependence than the Rouse modes. ${ }^{15}$ The $T_{\mathrm{g}}$ of the PS in ref 14 were determined by dilatometry with $q_{\mathrm{c}}=1 \mathrm{deg} / \mathrm{min}$ and conform to the equation

$$
T_{\mathrm{g}}=98.0-1.02 \times 10^{2} / M
$$

with $T_{\mathrm{g}}$ in ${ }^{\circ} \mathrm{C}$ and $M$ in $\mathrm{kDa}$. $T_{\mathrm{g}}$ values were also determined in ref 14 from the shift factors for the recoverable compliance, and these were in agreement with the dilatometric values. Equation 2 does not apply at very low $M$; thus, for A-67 with $M=1.1 \mathrm{kDa}, T_{\mathrm{g}}$ is taken to be $34{ }^{\circ} \mathrm{C}$, as explained in ref 14 . From the known $\tau_{\eta}(T)$ and $T_{\mathrm{g}}$ for each PS sample, $\log \tau_{\eta}(T)$ is plotted vs $T_{\mathrm{g}} / T$, with a vertical shift applied so that $\log \tau_{\eta}\left(T_{\mathrm{g}} / T\right)$ has a fixed, arbitrary value at $T_{\mathrm{g}} / T=1$. Most of the samples do not have data for $\tau_{\eta}\left(T_{\mathrm{g}} / T\right)$ exactly at $T_{\mathrm{g}} / T=1$, and hence the magnitudes of the vertical shifts in these cases are equivocal. With the important feature that $T_{\mathrm{g}}$ of all samples were determined by dilatometry at $q_{\mathrm{c}}=1 \mathrm{deg} / \mathrm{min}$, the results (Figure 1) show that the $T_{\mathrm{g}}$-normalized temperature dependences of $\tau_{\eta}$ for the PS are not exactly the same (albeit the differences are not large).

Measurements were also made on another sample of PS ( $M$ $=850 \mathrm{kDa})$, neat and in solutions of tritolyl phosphate (TCP). ${ }^{8,17}$ Viscosities were obtained only at temperatures sufficiently above $T_{\mathrm{g}}$ such that $J_{\mathrm{s}}$ is independent of $T$, and hence $\eta$ and $\tau_{\eta}$ have the same temperature dependence. We take the values of $T_{\mathrm{g}}$ determined by dilatometry (see upper curve of Figure 1 in ref 17) and plot in Figure $2 \log \eta$ as a function of $T_{\mathrm{g}} / T$ for 1.25 , $10,25,40,55$, and 70 wt $\%$ solutions of PS in TCP. Arbitrary vertical shifts have been applied to achieve overlap with the

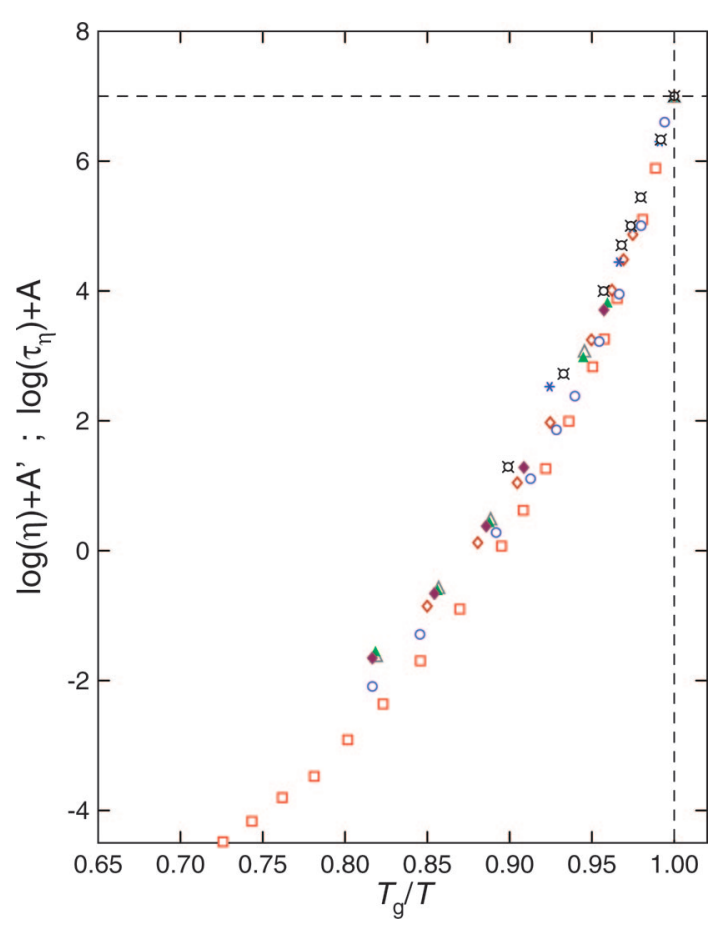

Figure 1. $T_{\mathrm{g}}$-normalized temperature dependence of $\log \tau_{\eta}(T)$ calculated by eq 1 of undiluted polystyrenes of various molecular weights, all determined by shear creep compliance and creep recovery measurements, with $T_{\mathrm{g}}$ from dilatometry at a cooling rate of $1 \mathrm{deg} / \mathrm{min}$. To allow comparison, vertical shifts have been applied to all data so that $\log \left[\tau_{\eta}\left(T_{\mathrm{g}} / T\right)\right]=7$ at $T_{\mathrm{g}} / T=1$. Open red squares (A67); black circles with four spokes $\left(\mathrm{A}-61^{3}\right)$; open red diamonds (A-25); open black triangles (A-19); open blue circles (PC11); closed blue stars (TAPS28); closed purple diamonds (M102); green plus signs (L2).

data for the undiluted PS at higher values of $T_{\mathrm{g}} / T$, taken from Figure 1. The deviations from superpositioning become larger with inclusion of the results for the PS solutions, particularly at lower values of $T_{\mathrm{g}} / T$.

b. Other Polymers. Similar creep compliance data are available for other amorphous polymers: poly(methyl methacrylate) (PMMA, $M=300 \mathrm{kDa}, T_{\mathrm{g}}=123^{\circ} \mathrm{C}$ from dilatometry at $q_{\mathrm{c}}=1 \mathrm{deg} / \mathrm{min}$ ), ${ }^{19}$ poly (vinyl acetate) (PVAc, $M=650 \mathrm{kDa}$, $T_{\mathrm{g}}=34.8^{\circ} \mathrm{C}$ from dilatometry at $q_{\mathrm{c}}=0.2 \mathrm{deg} / \mathrm{min}$ with a value of $36.8^{\circ} \mathrm{C}$ estimated for $\left.q_{\mathrm{c}}=1 \mathrm{deg} / \mathrm{min}\right),{ }^{12,20} \mathrm{PIB}\left(M_{\mathrm{w}}=78.5\right.$ $\mathrm{kDa}, T_{\mathrm{g}}=-76.0^{\circ} \mathrm{C}$ from dilatometry at $q_{\mathrm{c}}=0.2 \mathrm{deg} / \mathrm{min}$ and is estimated to be $-74.0{ }^{\circ} \mathrm{C}$ at $\left.q_{\mathrm{c}}=1 \mathrm{deg} / \mathrm{min}\right),{ }^{8,12}$ poly(methylphenylsiloxane) (PMPS, $M_{\mathrm{w}}=5 \mathrm{kDa}, T_{\mathrm{g}}=-49.7^{\circ} \mathrm{C}$ from DSC at $\left.q_{\mathrm{h}}=10 \mathrm{deg} / \mathrm{min}\right),{ }^{21}$ and $\mathrm{aPP}\left(M_{\mathrm{w}}=105 \mathrm{kDa}, T_{\mathrm{g}}\right.$ $=-11{ }^{\circ} \mathrm{C}$ from DSC at $\left.q_{\mathrm{h}}=10 \mathrm{deg} / \mathrm{min}\right),{ }^{5,6}$ these are shown in Figure 3. We compare but do not consider for the moment the PMPS or aPP because their $T_{\mathrm{g}}$ were not obtained by dilatometry.

The polymers PMMA, PVAc, and PIB all have high molecular weights with temperature-independent $J_{\mathrm{s}}$, so that the $T_{\mathrm{g}}$-normalized dependence of $\tau_{\eta}$ can be taken from $\eta\left(T_{\mathrm{g}} / T\right)$. The $T_{\mathrm{g}} \mathrm{S}$ used in Figure 3 polymers come from dilatometry at $q_{\mathrm{c}}=$ $1 \mathrm{deg} / \mathrm{min}$. The $\tau_{\eta}$ have been shifted vertically to achieve the best superposition with those of the undiluted PS, the results for which are reproduced from Figure 1. It is important to bear in mind that whatever vertical shifts are applied to the data the $T_{\mathrm{g}}$-normalized dependence of $\log \tau_{\eta}$ must either equal or smoothly extrapolate to the same predetermined value at $T_{\mathrm{g}} / T$ $=1$ (chosen to be 7 in all figures herein, but of course this statement is independent of the particular value used for $\tau_{\eta}\left(T_{\mathrm{g}} / T\right.$ $=1)$ ). For those cases where data are lacking for $\tau_{\eta}\left(T_{\mathrm{g}} / T\right)$ near $T_{\mathrm{g}} / T=1$, vertical shifts were made to superpose the data in the vicinity of the other polymers having similar curvature of $\tau_{\eta}\left(T_{\mathrm{g}} /\right.$ 


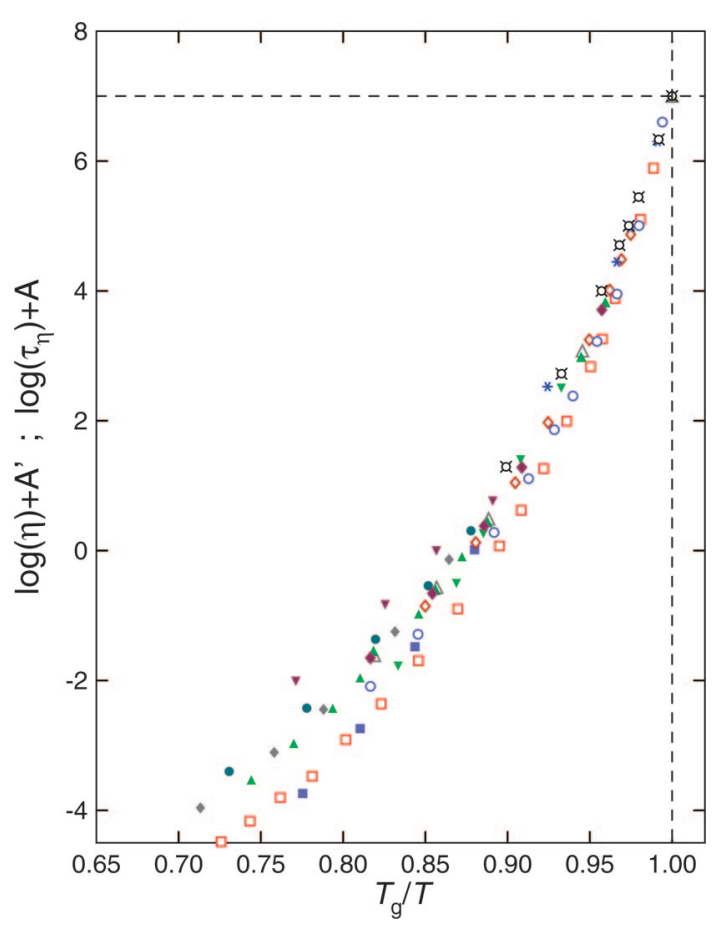

Figure 2. $T_{\mathrm{g}}$-normalized temperature dependence of $\log \eta(T)$ of PS $\left(M=8.5 \times 10^{5} \mathrm{Da}\right)$ and its solutions in TCP, together with the $T_{\mathrm{g}^{-}}$ normalized temperature dependence of $\log \tau_{\eta}(T)$ of undiluted polystyrenes from Figure 1. All determined by shear creep compliance and creep recovery measurements, with $T_{\mathrm{g}}$ from dilatometry at $q_{\mathrm{c}}=1 \mathrm{deg} /$ min. To allow comparison, vertical shifts have been applied to all data to make $\log \tau_{\eta}(T)$ and $\log \left[\tau_{\eta}\left(T_{\mathrm{g}} / T\right)\right]$ have the value of 7 at $T_{\mathrm{g}} / T=1$. Closed inverted green triangles (1.25 wt \% PS in TCP); closed blue squares (10\% PS); closed gray diamonds (25\% PS); closed magenta inverted triangles (40\% PS); closed blue circles (55\% PS); closed green triangles (70\% PS). For the undiluted PS, the symbols and colors are the same as in Figure 1.

T). It is evident by inspection that the data in Figure 3 do not support the existence of a universal $T_{\mathrm{g}}$-normalized temperature dependence of the chain dynamics.

The viscosity and the recoverable compliance shift factors for the PIB considered herein have nearly the same $T$ dependence down to about $-63^{\circ} \mathrm{C}$, the lowest temperature for which $\eta$ was measured. ${ }^{8}$ The shift factors for the recoverable compliance were determined down to 1 deg below $T_{\mathrm{g}}$ and support the observation that the $T_{\mathrm{g}}$-normalized temperature dependence is weaker for PIB than for PS and the other polymers. Note in Figure 3 that the viscosity data for PIB exhibit a slightly weaker dependence than does the recoverable compliance.

In further support of these results we include in Figure 3 (inverted triangles) dynamic mechanical terminal relaxation data of a polybutadiene, 1,2-PBD $\left(94 \mathrm{~mol} \%\right.$ vinyl content, $M_{\mathrm{w}}=$ $80.1 \mathrm{kDa}, T_{\mathrm{g}}=268.7 \mathrm{~K}$ ) reported by Robertson and Rademacher. ${ }^{29}$ The $T_{\mathrm{g}}$ were obtained by DSC at $q_{\mathrm{h}}=5 \mathrm{deg} / \mathrm{min}$ after cooling into the glassy state at the same rate. This calorimetric $T_{\mathrm{g}}$ of $1,2-\mathrm{PBD}$ is comparable to the value of $266.2{ }^{\circ} \mathrm{C}$ determined by dilatometry with a slightly smaller $q_{\mathrm{c}}=1 \mathrm{deg} /$ min on a similarly high vinyl polybutadiene. ${ }^{12}$ Thus, these data of 1,2-PBD can be included in Figure 3 for comparison with the other mechanical data, although the $T_{\mathrm{g}}$ for the latter are from dilatometry. The results for 1,2-PBD corroborate the absence of any universality of the $T_{\mathrm{g}}$-normalized temperature dependence of the terminal chain dynamics..

c. Tests for Polymers with $T_{\mathrm{g}}$ Determined by DSC. In testing the universality of chain dynamics in polymers, we pointed out that a requirement for critical assessment is uniformity in the determination of $T_{\mathrm{g}}$. The terminal chain

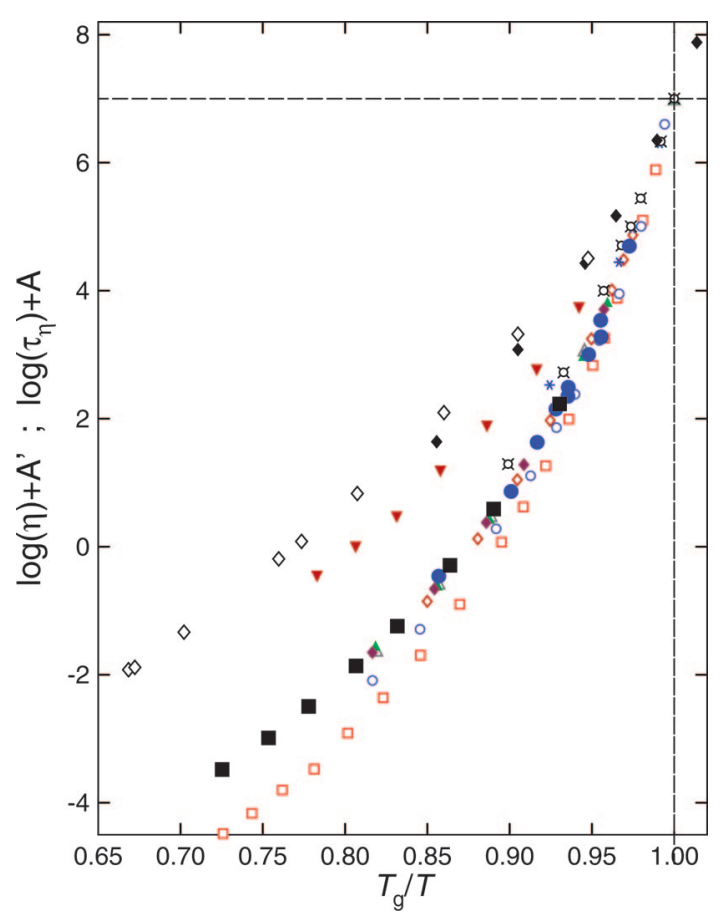

Figure 3. $T_{\mathrm{g}}$-normalized temperature dependence of $\log \eta(T)$ or $\log$ $\tau_{\eta}(T)$ of several amorphous polymers: $\mathrm{PIB}$ (uppermost two curves-viscosity, open green diamonds; shift factors for recoverable compliance, closed green diamonds); 1,2-PBD (immediately below PIB data-closed red inverted triangles); PVAc (closed black squares, located near the open red squares of PS A67); PMMA (closed brown circles near PVAc). The remaining symbols represent the $T_{\mathrm{g}}$-normalized temperature dependence of $\log \eta(T)$ or $\log \tau_{\eta}(T)$ of undiluted PS, with the symbols and colors as in Figure 1. All viscosities and retardation times were determined by shear creep compliance and creep recoverable measurements, with $T_{\mathrm{g}}$ from dilatometry at $q_{\mathrm{c}}=1 \mathrm{deg} / \mathrm{min}$.

dynamics of the $\mathrm{PMPS}^{21}$ and $\mathrm{aPP}^{5,6}$ in Figure 3 were obtained by creep measurements in the same laboratory of one of us; however, due inter alia to the limited amount of material, the $T_{\mathrm{g}}$ for these two polymers were determined by calorimetry, rather than by dilatometry; the cooling and heating rates were $10 \mathrm{deg} / \mathrm{min}$. Dilatometry and calorimetry can yield significantly different $T_{\mathrm{g}}$, and for both methods the value is dependent on the rate of temperature change. For high molecular weight polystyrene, DSC measurements at $q_{\mathrm{h}}=10 \mathrm{deg} / \mathrm{min}$ after cooling at the same rate yields $T_{\mathrm{g}}=105{ }^{\circ} \mathrm{C}$, as opposed to 98 ${ }^{\circ} \mathrm{C}$ from dilatometry at $q_{\mathrm{c}}=$ of $1 \mathrm{deg} / \mathrm{min}$ (see eq 2). The same trend was found in the low molecular weight PS sample A-67, with DSC giving $T_{\mathrm{g}}=40^{\circ} \mathrm{C}, 6 \mathrm{deg}$ higher than the value from dilatometry used in Figures 1-3. Since the two PS samples A-67 and A-19 are the extremes in both molecular weight and $T_{\mathrm{g}^{-}}$ normalized temperature dependence in Figure 1, we reconsider them using the calorimetric values of $T_{\mathrm{g}}=105^{\circ} \mathrm{C}$ for A-19 and $40{ }^{\circ} \mathrm{C}$ for A-67; Figure 4 includes the viscosity data of aPP and PMPS. For PMPS, $\tau_{\eta}$ was calculated as the product $\eta J_{\mathrm{s}}$ because $J_{\mathrm{S}}$ is $T$-dependent. ${ }^{21}$ Also included are two polybutadienes, 1,4-PBD (7 mol \% vinyl content, $M=38.7 \mathrm{kDa}, T_{\mathrm{g}}$ $=180.8 \mathrm{~K}$ ) and the $1,2-\mathrm{PBD}^{29}$ from Figure 3 . It is well-known that $T_{\mathrm{g}}$ increases with increase in cooling and heating rate, implying that $T_{\mathrm{g}}$ of these polybutadienes would be even higher than the values used herein. This means that the $T_{\mathrm{g}}$-normalized dependences of the viscosity of 1,4-PBD and 1,2-PBD in Figure 4 would be flatter and deviate further from the results for the other polymers. It can be concluded from Figure 4 that the terminal polymer chain dynamics determined by mechanical measurements using calorimetric $T_{\mathrm{g}}$ at a constant $q$ are not universal. 


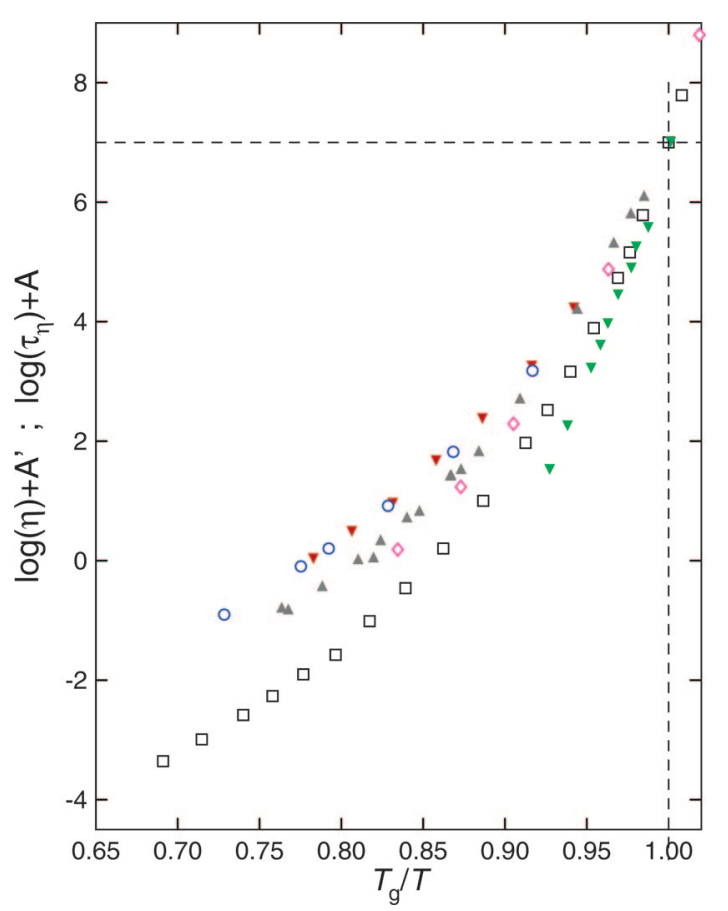

Figure 4. $T_{\mathrm{g}}$-normalized temperature dependence of $\log \eta(T)$ or $\log$ $\tau_{\eta}(T)$ of several amorphous polymers, with $T_{\mathrm{g}}$ from dilatometry at rates of $10 \mathrm{deg} / \mathrm{min}$. PMPS (closed inverted green triangles); aPP (closed gray triangles); 1,2-PBD (closed red inverted triangles); 1,4-PBD (open blue circles); PS A-67 (open red squares); PS A-19 (open magenta diamonds).

\section{Limitation of Using Dielectric Relaxation Data}

A few polymers such as PI, PPG, and POB have a dipole moment parallel to the chain backbone, giving rise to a normal mode peak in the dielectric loss. The terminal chain relaxation time $\tau_{\mathrm{n}}$ can be obtained from the (inverse) frequency of this loss peak. The POB data from dielectric relaxation examined here are the same as used by Ding and Sokolov (DS) ${ }^{1}$ and come from Casalini and Roland. ${ }^{3}$ Dielectric data for PPG $(M=4.00$ $\mathrm{kDa})$ and PI $(M=1.23 \mathrm{kDa})$ are taken from refs 30 and 31 , respectively. The $T_{\mathrm{g}}$ of POB, PPG, and PI, $-74.3,-75.8$, and $-79.2{ }^{\circ} \mathrm{C}$, respectively, are defined as the temperature corresponding to a fixed value of $\tau_{\alpha}\left(T_{\mathrm{g}}\right)=100 \mathrm{~s}$, where $\tau_{\alpha}$ is the dielectric $\alpha$-relaxation time. In contrast, DS chose $\tau_{\alpha}\left(T_{\mathrm{g}}\right)=1 \mathrm{~s}$ to define their $T_{\mathrm{g}}$, a less demanding choice because, as stated in the Introduction, a shorter segmental relaxation time moves the focus away from $T_{\mathrm{g}}$ where $T$ sensitivities are strongest. $\mathrm{DS}^{1}$ combined the dielectric normal mode data for POB, PPG, and PI data with viscosity data of other polymers to test the universality of $T_{\mathrm{g}}$-normalized temperature dependence of the terminal chain dynamics. This comparison was done in ref 1 using for high molecular weight PS $T_{\mathrm{g}}=100{ }^{\circ} \mathrm{C}$, rather than the published dilatometric value ${ }^{14}$ (used herein in Figure 1), and for aPP $T_{\mathrm{g}} \sim-6{ }^{\circ} \mathrm{C}$ rather than the published DSC value ${ }^{5}$ of $-11{ }^{\circ} \mathrm{C}$ (used herein in Figure 3). $\alpha$-Relaxation times determined by dielectric vs mechanical relaxation can differ greatly, ${ }^{32}$ and hence the corresponding $T_{\mathrm{g}}$ values will differ. The point is that mixing dielectric and mechanical results introduces uncertainties into a test of the universality of chain dynamics. Nevertheless, following ref 1, we compare in Figure 5 the $T_{\mathrm{g}}$-normalized temperature dependence of the dielectric $\tau_{\mathrm{n}}$ of POB, PI, and PPG using $\tau_{\alpha}\left(T_{\mathrm{g}}\right)=100 \mathrm{~s}$ with creep data for high molecular weight PS, A-61, A-25, and L2 using the $T_{\mathrm{g}}$ from ref 14 . These are the same PS data used by DS. ${ }^{1}$ For POB, $\tau_{\mathrm{n}}$ (closed circles) extend to $T_{\mathrm{g}} / T$ as high as 0.99 , so that our requirement that $\log \tau_{\mathrm{n}}=7$ at $T_{\mathrm{g}}$ is satisfied. The $T_{\mathrm{g}}$-normalized

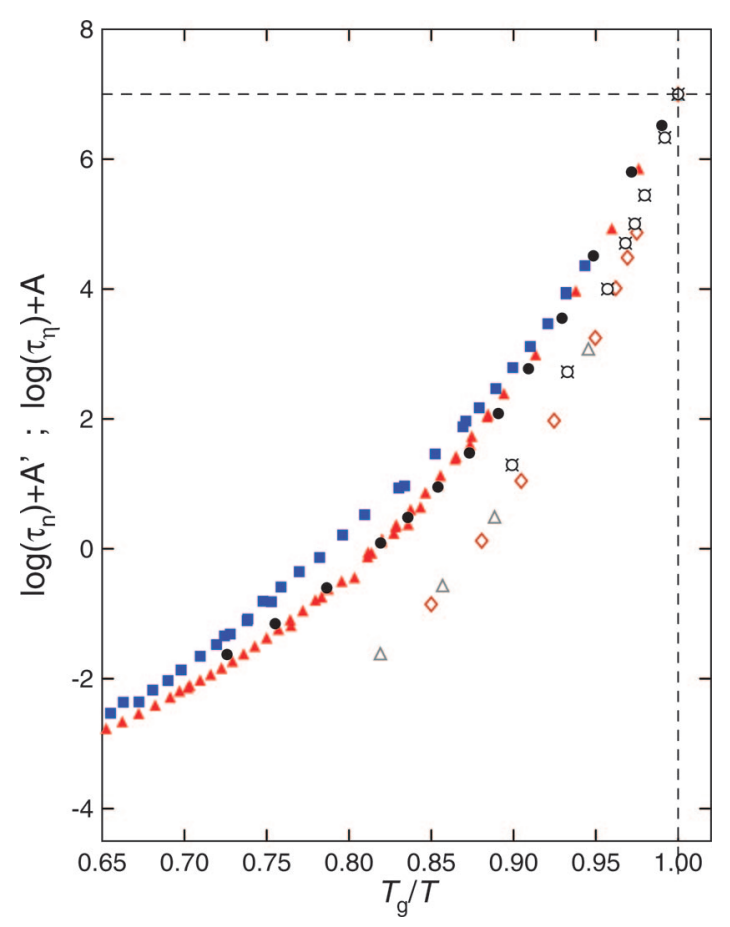

Figure 5. $T_{\mathrm{g}}$-normalized temperature dependence of $\log \tau_{\mathrm{n}}(T)$ from dielectric relaxation measurements of PI (closed blue squares), POB (closed black circles), and PPG4000 (closed red triangles). Shown also are $T_{\mathrm{g}}$-normalized temperature dependences of $\log \eta(T)$ or $\log \tau_{\eta}(T)$ of undiluted PS A-19 (open gray triangles), A-25 (open red diamonds), and A-61 (open circles with four spokes outside) from Figure 1.

temperature dependence of $\tau_{\mathrm{n}}$ for PPG (closed triangles) and PI (closed squares) are not much different from that of POB. This is in accord with the observation of Sokolov and coworkers $^{1,2}$ for the dielectric relaxation data of these three polymers. However, when the creep compliance data of PS are included in Figure 5, any universality is lost. The $T_{\mathrm{g}}$-normalized temperature dependences of $\tau_{\mathrm{n}}$ and $\tau_{\eta}$ are clearly different. Note that using $\tau_{\alpha}\left(T_{\mathrm{g}}\right)=1$ or $10 \mathrm{~s}$ as done by Sokolov and co-workers, ${ }^{1,2}$ rather than $\tau_{\alpha}\left(T_{\mathrm{g}}\right)=100 \mathrm{~s}$ here, would weaken the $T_{\mathrm{g}}$-normalized temperature dependence of $\tau_{\mathrm{n}}$ in Figure 5 , increasing the differences with the other polymers. An obvious question is why for the same PS do we reach a conclusion herein opposite to that of DS ${ }^{1}$ We can only speculate that while the PS viscosities in Figure 5 were taken from the tabulated values in ref 14 , DS cite ref 8 as their source, which would require extracting values from Figure 4 therein. The latter procedure is less accurate.

From the results compiled by Kremer, ${ }^{33} \tau_{\mathrm{n}}$ for PI has the $M^{2}$ dependence of Rouse behavior expected for $M$ up to at least 10 $\mathrm{kDa}$. Therefore, for the PI samples considered herein and studied by Sokolov and co-workers, , 2 the normal modes conform to Rouse dynamics. This means that any conclusion about the universality of the $T_{\mathrm{g}}$-normalized temperature dependence of $\tau_{\mathrm{n}}$ does not extend to entangled PI. In fact, dielectric relaxation measurements will always be inadequate to address the terminal dynamics of high molecular weight polymers due to the large separation of $\tau_{\mathrm{n}}$ from $\tau_{\alpha}$. This is apparent in the dielectric data of Sokolov and Hayashi, ${ }^{2}$ in which even for unentangled PI with $M=9.55 \times 10^{3} \mathrm{Da}, \tau_{\mathrm{n}}$ can only be determined for $T_{\mathrm{g}} / T$ smaller than about 0.95 where $T_{\mathrm{g}}$ is defined by $\tau_{\alpha}\left(T_{\mathrm{g}}\right)=1 \mathrm{~s}$. Transient compliance measurements are necessary to measure $\tau_{\eta}$ for entangled PI sufficiently close to $T_{\mathrm{g}}$ and thereby allow critical assessment of the universality of chain dynamics.

Sokolov and co-workers ${ }^{1,2}$ also used viscosity data of polycarbonate with $M_{\mathrm{w}}=32 \mathrm{kDa}$ from ref 10 to support their contention of universal behavior. These viscosity data were 
obtained by an unconventional technique - the penetration depth of a punch into the sample surface. ${ }^{10}$ Such a test does not maintain constant stress, constant strain rate, or a homogeneous state of strain; hence, the results are only qualitatively reliable. This can be seen by considering that at $T=154{ }^{\circ} \mathrm{C}$ (the $T_{\mathrm{g}}$ in ref 35 and used by Sokolov and co-workers ${ }^{1,2}$ ) the viscosity reported in ref 10 is $\sim 2 \times 10^{9} \mathrm{~Pa}$ s. The plateau modulus $G_{\mathrm{e}}$ of $\mathrm{PC}$ is $2.7 \mathrm{MPa},{ }^{34}$ and hence the terminal relaxation time $\tau_{\eta}$ estimated from the Maxwell relation, $\tau_{\eta}=\eta / G_{\mathrm{e}}$, is about $10^{3} \mathrm{~s}$. The entanglement molecular weight $M_{\mathrm{e}}$ of PC is $1.66 \mathrm{kDa},{ }^{34}$ which means the PC sample in question is highly entangled. At $T_{\mathrm{g}}, \tau_{\alpha}$ is of the order of $100 \mathrm{~s}$, and $\tau_{\eta}$ must be many orders of magnitude larger, as reflected in the extended length of the rubbery plateau (due to the $M^{3.4}$ dependence of $\tau_{\eta}$ and $\eta$ for entangled polymers). Accordingly, the viscosity of the PC cannot be as low as $2 \times 10^{9} \mathrm{~Pa}$ s at $T_{\mathrm{g}}$. Note for comparison, for many nonpolymeric glass-formers, $\eta$ at $T_{\mathrm{g}}$ is about $10^{12} \mathrm{~Pa}$ s, or 3 orders of magnitude higher than reported 10 for entangled PC at $T_{\mathrm{g}}$. Given the obvious problems with the PC viscosity data of PC in ref 10 it cannot be used to support a claim of universal chain dynamics.

\section{Conclusion}

In assessing the universality of the chain dynamics of polymers suggested in refs 1,2 , and 11 , we emphasize the importance of (i) using terminal relaxation data obtained by the same technique, (ii) measured close to $T_{\mathrm{g}}$, and (iii) analyzing with a uniformly determined $T_{\mathrm{g}}$. In this work we utilized a large body of viscoelastic data obtained by creep and recoverable compliance measurements in a single laboratory, with $T_{\mathrm{g}}$ from either dilatometry or calorimetry. The results show clear deviations from a universal $\tau_{\eta}\left(T_{\mathrm{g}} / T\right)$ behavior. Additional data, for example from modulus or dielectric relaxation experiments, corroborate the breakdown of universality in the $T_{\mathrm{g}}$-normalized temperature dependence of the terminal (normal mode) relaxation time of polymers. Nevertheless, the $T_{\mathrm{g}}$-normalized temperature dependence of the terminal relaxation time of polystyrenes with molecular weights ranging from $10^{3}$ to more than 1 million Da are intriguingly similar. This is an interesting result that will be addressed theoretically in a future publication.

Acknowledgment. The research at NRL was supported by the Office of Naval Research. Discussions with A. P. Sokolov are gratefully acknowledged.

\section{References and Notes}

(1) Ding, Y.; Sokolov, A. P. Macromolecules 2006, 39, 3322.

(2) Sokolov, A. P.; Hayashi, Y. J. Non-Cryst. Solids 2007, 353, 3838.

(3) Casalini, R.; Roland, C. M. Macromolecules 2005, 38, 1779.
(4) Ngai, K. L.; Casalini, R.; Roland, C. M. Macromolecules 2005, 38, 4363.

(5) Plazek, D. L.; Plazek, D. J. Macromolecules 1983, 16, 1569.

(6) Roland, C. M.; Ngai, K. L.; Santangelo, P. G.; Qiu, X. H.; Ediger, M. D.; Plazek, D. J. Macromolecules 2001, 34, 6159.

(7) Ferri, D.; Castellani, L. Macromolecules 2001, 34, 3973.

(8) Plazek, D. J.; Zheng, X. D.; Ngai, K. L. Macromolecules 1992, 25, 4920.

(9) Liu, C.; Li, C.; Chen, P.; He, J.; Fan, Q. Polymer 2004, 45, 2803.

(10) Yang, F. Polym. Eng. Sci. 1997, 37, 101.

(11) Liu, C. Y.; He, J. S.; Keunings, R.; Bailly, C. Macromolecules 2006, $39,8867$.

(12) Plazek, D. J.; Ngai, K. L. In Physical Properties of Polymers Handbook, 2nd ed.; Mark, J. E., Ed.; Springer: Berlin, 2007; Chapter 12.

(13) Roland, C. M.; Casalini, R. J. Chem. Phys. 2003, 119, 1838.

(14) Plazek, D. J.; O'Rourke, V. M. J. Polym. Sci., Part A-2 1971, 9, 209.

(15) Ngai, K. L.; Plazek, D. J. Rubber Chem. Technol. 1995, 68, 376.

(16) Plazek, D. J.; Raghupathi, N.; Orbon, S. J. J. Rheol. 1979, 23, 477.

(17) Riande, E.; Markovitz, H.; Plazek, D. j.; Raghupathi, N. J. Polym. Sci., Symp. 1975, 50, 405. The cooling rate in the dilatometry experiments was $1 \mathrm{deg} / \mathrm{min}$, and not $1 \mathrm{deg} / \mathrm{h}$ as erroneously stated in this reference.

(18) Plazek, D. J.; Riande, E.; Markovitz, H.; Raghupathi, N. J. Polym. Sci., Polym. Phys. Ed. 1979, 17, 2189.

(19) Plazek, D. J.; Tan, V.; O'Rourke, V. M. Rheol. Acta 1974, 13, 367. The dilatometric $T_{\mathrm{g}}$ determined at $q_{\mathrm{c}}=1 \mathrm{deg} / \mathrm{h}$ is $117^{\circ} \mathrm{C}$ (see also ref 12). From the known dependence of the dilatometric $T_{\mathrm{g}}$ on $q_{\mathrm{c}}$ for PS from Greiner and Schwartzl (see ref 12), there is an increase of about 4 and $6 \mathrm{deg}$ for respective cooling rates of 0.2 and $1 \mathrm{deg} / \mathrm{min}$. We use these to obtain 121 and $123{ }^{\circ} \mathrm{C}$ as the dilatometric $T_{\mathrm{g}}$ of PMMA at $q_{\mathrm{c}}=0.2$ and $1 \mathrm{deg} / \mathrm{min}$, respectively.

(20) Plazek, D. J. Polym. J. 1980, 12, 43.

(21) Plazek, D. J.; Bero, C.; Neumeister, S.; Floudas, G.; Fytas, G.; Ngai, K. L. J. Colloid Polym. Sci. 1994, 272, 1430.

(22) Ngai, K. L.; Plazek, D. J.; Bero, C. Macromolecules 1993, 26, 1065.

(23) Plazek, D. J.; Chay, I.-C.; Ngai, K. L.; Roland, C. M. Macromolecules $1995,28,6432$.

(24) Ngai, K. L.; Plazek, D. J.; Rizos, A. K. J. Polym. Sci., Part B: Polym. Phys 1997, 35, 599.

(25) Plazek, D. J.; Schlosser, E.; Schönhals, A.; Ngai, K. L. J. Chem. Phys. 1993, 98, 6488.

(26) Ferry, J. D. Viscoelastic Properties of Polymers, 3rd ed.; John Wiley \& Sons: New York, 1980.

(27) Note that eq 1 is sometimes written to include a constant prefactor that does not affect the $T$ dependence [see ref 26].

(28) Santangelo, P. G.; Roland, C. M. J. Rheol. 2001, 45, 583.

(29) Robertson, C. G.; Rademacher, C. M. Macromolecules 2004, 37, 10009.

(30) Ngai, K. L.; Schönhals, A.; Schlosser, Macromolecules 1992, 25, 4915.

(31) Schönhals, A. Macromolecules 1993, 26, 1309.

(32) McCrum, N. G.; Read, B. E.; Williams, G. Anelastic and Dielectric Effects in Polymer Solids; Wiley: London, 1967.

(33) Kremer, F. In Physical Properties of Polymers Handbook, 2nd ed.; Mark, J. E., Ed.; Springer: Berlin, 2007; Chapter 21.

(34) Fetter, L. J.; Lohse, D. J.; Colby, R. H. In Physical Properties of Polymers Handbook, 2nd ed.; Mark, J. E., Ed.; Springer: Berlin, 2007; Chapter 25.

MA702814G 